\title{
Early menopause and premature ovarian insufficiency are associated with increased risk of type 2 diabetes: a systematic review and meta-analysis
}

\author{
Panagiotis Anagnostis 1,2,3, Konstantinos Christou ${ }^{2,3}$, Aikaterini-Maria Artzouchaltzi ${ }^{2,3}$, Nifon K Gkekas ${ }^{2,3}$, \\ Nikoletta Kosmidou, 2,3, Pavlos Siolos ${ }^{2,3}$, Stavroula A Paschou ${ }^{4}$, Michael Potoupnis ${ }^{3,5}$, Eustathios Kenanidis ${ }^{3,5}$, \\ Eleftherios Tsiridis ${ }^{3,5}$, Irene Lambrinoudaki ${ }^{6}$, John C Stevenson? ${ }^{7}$ and Dimitrios G Goulis ${ }^{1,3}$
}

${ }^{1}$ First Department of Obstetrics and Gynecology, Unit of Reproductive Endocrinology, Medical School, Aristotle University of Thessaloniki, Thessaloniki, Greece, ${ }^{2}$ Police Medical Center of Thessaloniki, Thessaloniki, Greece, ${ }^{3} \mathrm{Centre}$ of Orthopedic and Regenerative Medicine Research (CORE), Center for Interdisciplinary Research and Innovation (CIRI), Aristotle University of Thessaloniki, Thessaloniki, Greece, “Division of Endocrinology and Diabetes, 'Aghia Sophia' Hospital, Medical School, National and Kapodistrian University of Athens, Athens, Greece, ${ }^{5}$ Academic Orthopaedic Unit, Aristotle University Medical School, General Hospital Papageorgiou, Thessaloniki, Greece, ${ }^{6}$ Second Department of Obstetrics and Gynecology, National and Kapodistrian University of Athens, Athens, Greece, and ${ }^{7}$ National Heart and Lung Institute, Imperial College London, Royal Brompton and Harefield NHS Foundation Trust, London, UK

Correspondence should be addressed to P Anagnostis Email anagnwstis.pan@yahoo.gr

\begin{abstract}
Objective/Design: Menopausal transition has been associated with a derangement of glucose metabolism. However, it is not known if early menopause (EM, defined as age at menopause $<45$ years) or premature ovarian insufficiency (POI, defined as age at menopause $<40$ years) are associated with increased risk of type 2 diabetes mellitus (T2DM). To systematically investigate and meta-analyze the best evidence regarding the association of age at menopause with the risk of T2DM.

Methods: A comprehensive search was conducted in PubMed, CENTRAL and Scopus, up to January 31, 2018. Data are expressed as odds ratio (OR) with 95\% confidence intervals (CI). The $R^{2}$ index was employed for heterogeneity. Results: Thirteen studies were included in the qualitative and quantitative analysis (191 762 postmenopausal women, 21664 cases with T2DM). Both women with EM and POI were at higher risk of T2DM compared with those of age at menopause of $45-55$ years (OR: 1.15, 95\% Cl: 1.04-1.26, $P=0.003 ; I^{2}: 61 \%, P<0.002$ and OR: $1.50,95 \%$ Cl: 1.03-2.19, $\left.P=0.033 ; I^{2}: 75.2 \%, P<0.003\right)$, respectively). Similar associations emerged when women with EM and POI were compared with those of age at menopause $>45$ years (OR: $1.12,95 \% \mathrm{Cl}: 1.01-1.20, P<0.02 ; I^{2}: 78 \%, P<0.001$ and OR: $\left.1.53,95 \% \mathrm{Cl}: 1.03-2.27, P=0.035 ; 1^{2}: 78 \%, P<0.001\right)$, respectively).
\end{abstract}

Conclusions: Both EM and POI are associated with increased risk of T2DM.

\section{Introduction}

Menopause is chronically determined by the completion of 12 months after the final menstrual period. It is the consequence follicular depletion leading to estrogen deficiency (1). The average age at menopause is $50-52$ years (2). However, approximately $10 \%$ of the female population enter menopause before 45 years, a condition termed 'early' or 'premature' menopause (3). About $1 \%$ of women enter menopause under the age of 40 ( $0.1 \%$ under the age of 30$)$, a condition termed 'premature ovarian insufficiency' (POI) $(3,4)$. Except the vasomotor
Published by Bioscientifica Ltd. 
symptoms affecting the woman's quality of life, transition to menopause has also been associated with a potential higher cardiovascular (CVD) risk, mainly attributed to a more atherogenic lipid profile, central adiposity and glucose intolerance $(5,6,7,8)$.

Both early menopause (EM) and POI have been associated with increased risk of death and CVD in recent meta-analyses $(9,10)$. With respect to glucose metabolism, the true effect of endogenous hormonal milieu during menopause on T2DM risk is controversial, since some, but not all, studies have shown a possible association between EM and increased risk of T2DM $(11,12,13,14)$. The association of T2DM with increased CVD risk has been well documented in both genders, although women with T2DM seem to be at a higher relative CVD risk compared with their male counterparts (15). However, whether EM or POI per se is associated with increased risk of T2DM is unknown.

The aim of this study was to systematically investigate and meta-analyze the best evidence regarding the association of menopausal age with the risk of developing T2DM.

\section{Subjects and methods}

\section{Guidelines followed}

This systematic review followed the PRISMA (Preferred Reporting Items for Systematic reviews and MetaAnalyses) guidelines (16). A flow diagram is available in Fig. 1. A completed PRISMA checklist is available as Supplementary Table 1 (see section on supplementary data given at the end of this article).

\section{Search strategy}

The following PICO (Population, Intervention or exposure, Comparison, Outcome) elements were applied as inclusion criteria for the systematic review: (i) Population: postmenopausal women; (ii) Intervention: early age at menopause, either EM or POI; (iii) Comparison group: women with natural menopause; (iv) Outcome: T2DM. To identify eligible studies, the main search was conducted in the electronic databases MEDLINE, Scopus and Cochrane (CENTRAL) covering the period from conception until January 31, 2018, and using the following search strings:

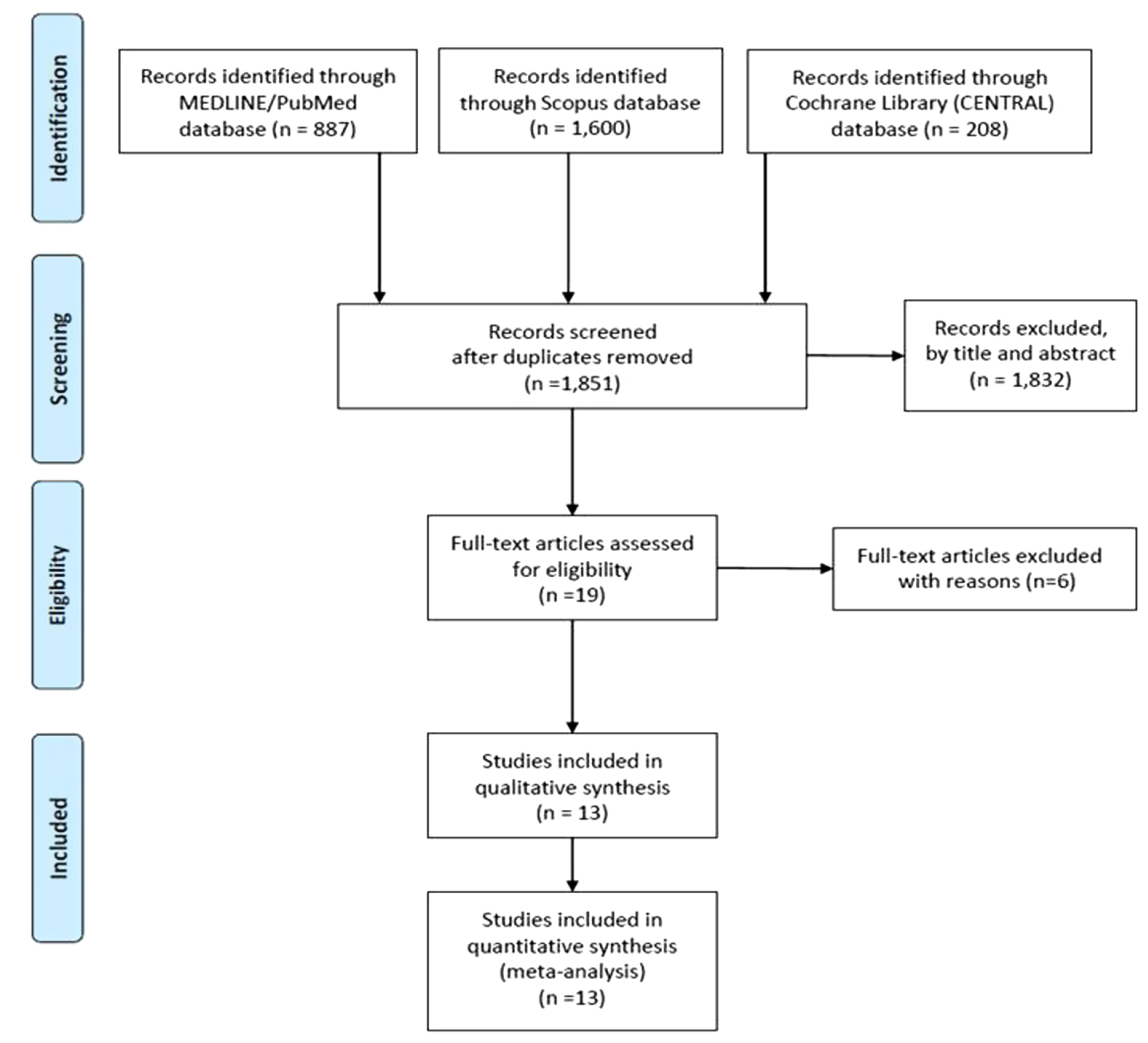

\section{Figure 1}

Flow chart diagram. 
("menopause, premature"[MeSH] OR "primary ovarian insufficiency"[MeSH] OR "ovarian insufficiency"[tiab] OR "ovarian failure"[tiab] OR ((menopause[MeSH] OR menopause[tiab] OR menopausal[tiab] OR climacteric[tiab] OR postmenopausal[tiab] OR post-menopausal[tiab]) AND (early[tiab] OR premature[tiab] OR age[tiab] OR years[tiab] OR time[tiab]))) AND ("diabetes mellitus, Type 2"[MeSH] OR (diabet*[tiab] AND ("non-insulin dependent"[tiab] OR "non-insulin-dependent"[tiab] OR type-2[tiab] OR "type 2"[tiab] OR "type II"[tiab])) OR diabetes[ti] OR diabetic[ti]) NOT (Animal[MeSH] NOT Human[MeSH]) NOT (letter[pt] OR comment[pt] OR editorial[pt] OR Review[pt] OR "practice guideline"[ptyp] OR "case reports"[ptyp]). The main search was completed independently by two investigators ( $\mathrm{K} \mathrm{C}$ and A M A). Any discrepancy was solved by consultation of an investigator, not involved in the initial procedure (P A and D G G).

\section{Trial selection}

Specific inclusion criteria were set as follows: (i) Studies conducted in postmenopausal women (either hysterectomized or non-hysterectomized) and (ii) studies providing extractable data. Both cohorts and case-control studies were eligible. Studies were excluded if they (i) Had no control group (without T2DM); (ii) Their population included pre- or peri-menopausal women; (iii) Included subjects receiving concomitant therapy with drugs affecting glucose metabolism, such as glucocorticoids; (iv) Were written in a language other than English; (v) Included patients with genetic syndromes associated with EM or POI (e.g. Turner's syndrome); (vi) Included women with a history of polycystic ovarian syndrome (PCOS) and (vii) Were conducted in animals.

\section{Data extraction}

Two researchers ( $\mathrm{K} \mathrm{C}$ and $\mathrm{A} \mathrm{M} \mathrm{A}$ ) reviewed all eligible studies. The following data were extracted and recorded: (i) First author; (ii) Year of publication; (iii) Country in which the study was conducted; (iv) Study design (casecontrol or cohort); (iv) Duration (available in cohorts); (v) Total number of participants; (vi) Number of women with EM; (vii) Number of women with POI; (viii) Number of women with normal menopause (subdivided in those with menopausal age between 45 and 55 years and those with a menopausal age $>45$ years; (ix) Number of women with late menopause (>55 years); (x) Number of cases with T2DM in each of these categories. Parameters such as mean age of the participants at study entry, mean BMI, the percentage of women with surgical menopause, the method of T2DM diagnosis, smoking status and physical activity were also recorded.

The following comparisons were made according to the incidence or prevalence of T2DM: (i) Women with EM compared with those with menopausal age of $>45$ years; (ii) Women with EM compared with those with menopausal age of 45-55 years; (iii) Women with POI compared with those with menopausal age of $>45$ years; (iv) Women with POI compared with those with menopausal age of 45-55 years; (v) Women with late menopause compared with those with menopausal age of 45-55 years.

\section{Risk of bias and study quality assessment}

Newcastle-Ottawa scale (NOS) was used for assessing the quality of each study. Briefly, this system evaluates studies based on three criteria: (i) Participant selection; (ii) Comparability of study groups and (iii) Assessment of outcome or exposure. A study can be awarded a maximum of four stars for the selection category, a maximum of two stars for the comparability category and a maximum of three stars for the outcome/exposure category (17). These results are available in Supplementary Table 2.

\section{Statistical analyses}

Heterogeneity was tested with the Cochrane chi-square test and the degree of heterogeneity was quantified by the $I^{2}$ statistics. An $I^{2}$ of $30-60 \%$ was considered as moderate, whereas values $>60 \%$ were considered as high degree of heterogeneity. Random effects model was used for data synthesis. Associations were reported as odds ratios (OR) with their 95\% confidence intervals (CIs). A $P$ value of $<0.05$ was considered as statistically significant. Publication bias was formally tested with Begg-Mazumdar test (presented in funnel plot diagram, with $P$ values $>0.1$ indicating absence of publication bias) and the Egger's test ( $P$ values $>0.1$ indicating absence of publication bias). All analyses were done with the software Comprehensive MetaAnalysis V2.

\section{Results}

\section{Descriptive data}

The initial search provided 1851 results, after excluding duplicates, 19 of which were assessed as full texts for eligibility (Fig. 1). Of those, six articles were excluded, due to the following reasons: (i) No data on T2DM according 
to menopausal age $(n=1)$; (ii) No data on EM $(n=3)$; (iii) Non-English language $(n=1)$; (iv) Referral to another paper already included in the analysis $(n=1)$. Thirteen studies were included in the qualitative and quantitative analysis $(11,12,13,14,18,19,20,21,22,23,24,25,26)$. The excluded studies and the reasons for their exclusion are available in Supplementary Table 3. The studies were published between 2013 and 2017. The countries in which they were conducted were China $(n=5)$, USA $(n=2)$, India $(n=1)$, Japan $(n=2)$, Vietnam $(n=1)$ and Netherlands $(n=1)$. One study was conducted in more than one country (in Europe). The number of participants ranged from 100 to 124379 , yielding a total number of 191762 postmenopausal women, with 21664 cases of T2DM. The mean age of participants was $62.6 \pm 7.6$ years (data available from nine studies) and mean BMI was $23.9 \pm 3.8 \mathrm{~kg} / \mathrm{m}^{2}$ (data available for seven studies).

Diagnosis of T2DM was set by various methods. Fasting plasma glucose (FPG) $\geq 126 \mathrm{mg} / \mathrm{dL}$ ( $7 \mathrm{mmol} / \mathrm{L}$ ) or glycated hemoglobin $\left(\mathrm{HbA}_{1 \mathrm{c}}\right) \geq 6.5 \%$ or plasma glucose $\geq 200 \mathrm{mg} / \mathrm{dL}(11.1 \mathrm{mmol} / \mathrm{L})$ at $2 \mathrm{~h}$, after an oral glucose tolerance test (OGTT) were used in eight studies $(12,14$, $18,21,22,24,25,26)$. Of note, in one study OGTT was performed with a glucometer (20) and in another study the method of T2DM diagnosis was not defined (13). In the remaining studies, the diagnosis was derived from self-reports, health care reports, hospital admissions or history of anti-diabetic medication use $(11,19,23)$. With respect to the type of menopause, five studies included only women with natural menopause $(12,13,24,25$, 26), five included mixed populations (9-39\% of which with surgical menopause) $(11,14,18,19,23)$ and in three studies $(20,21,22)$ the type of menopause was not clearly defined. No data on prediabetes (defined either as 'impaired fasting glucose' or 'impaired glucose tolerance') were available. The descriptive characteristics of the studies' participants are presented in Tables 1 and 2.

\section{Comparison of women with EM with those with normal menopausal age}

Women with EM displayed a higher risk of developing T2DM compared with women with normal menopausal age ( $>45$ years) including also those with late menopausal age (12 studies, four prospective cohorts) $(11,12,13$, 14, 18, 19, 21, 22, 23, 24, 25, 26) (OR: 1.12, 95\% CI: 1.01-1.23, $\left.P=0.019 ; I^{2}: 65.69 \%, P<0.001\right)$. One study did not provide absolute number of participants in both T2DM and non-T2DM groups and, therefore, OR was directly included in the analysis (20) (Fig. 2).

Compared with women with menopausal age of 45-55 years, women with EM were also at a higher risk for T2DM (13 studies, four prospective cohorts) (OR: 1.15, 95\% CI: $\left.1.04-1.26, P=0.003 ; I^{2}: 61 \%, P<0.002\right)(11,12,13$, 14, 18, 19, 20, 21, 22, 23, 24, 25, 26) (Fig. 3). A variation with respect to the upper limit of normal menopausal age must be noted (ranging from 49 to 55, mostly 52-55). After excluding three studies having used the age of 49 or 50 years as the upper limit of normal menopausal age, the OR of the remaining studies remained significant (1.13, 95\% CI: 1.03-1.24, $P=0.008)(13,18,20)$.

Table 1 Demographic characteristics of studies included in the analysis.

\begin{tabular}{|c|c|c|c|c|c|c|c|c|c|}
\hline \multirow[b]{2}{*}{ Study } & \multirow[b]{2}{*}{ Study design } & \multicolumn{6}{|c|}{ Characteristics of the participants } & \multicolumn{2}{|c|}{ Type of menopause } \\
\hline & & $\begin{array}{c}\text { Total } \\
\text { number }\end{array}$ & $\begin{array}{c}\text { Women } \\
\text { with T2DM } \\
\end{array}$ & $\begin{array}{c}\text { Use of HRT, } \\
n(\%) \\
\end{array}$ & Use of OC, $n(\%)$ & Mean age (years) & $\mathrm{BMI}\left(\mathrm{kg} / \mathrm{m}^{2}\right)$ & Natural, $n(\%)$ & Other, $n(\%)$ \\
\hline$(13)$ & Case-Control & 100 & 6 & N/A & N/A & $\mathrm{N} / \mathrm{A}$ & $\mathrm{N} / \mathrm{A}$ & $100(100)$ & $0(0)$ \\
\hline (11) & Cohort & 7864 & 3691 & $1292(29)^{*}$ & $1792(40)^{*}$ & $59.2 \pm 5.8$ & $26.3 \pm 4.6$ & $3550(78)^{*}$ & $858(22)^{\star}$ \\
\hline$(12)$ & Case-Control & 4318 & 140 & $603(14)$ & $\mathrm{N} / \mathrm{A}$ & $53.5 \pm 3.3$ & $22.6 \pm 2.8$ & $4318(100)$ & $0(0)$ \\
\hline (14) & Case-Control & 3304 & 738 & N/A & N/A & $59.4 \pm 8.2$ & $24.3 \pm 3.2$ & $3003(90)$ & $301(10)$ \\
\hline$(18)$ & Case-Control & 4416 & 170 & N/A & N/A & $57.1 \pm 6.6$ & $21.8 \pm 3.0$ & 3552 (78) & $1018(22)$ \\
\hline (19) & Cohort & 2597 & 176 & 797 (30) & $532(20)$ & $60.7 \pm 10.7$ & N/A & $1562(60)$ & 1035 (40) \\
\hline$(20)$ & Case-Control & 608 & 46 & N/A & N/A & $\mathrm{N} / \mathrm{A}$ & N/A & $\mathrm{N} / \mathrm{A}$ & N/A \\
\hline$(21)$ & Case-Control & 2099 & 394 & N/A & N/A & $66.1 \pm 4.8$ & N/A & $\mathrm{N} / \mathrm{A}$ & N/A \\
\hline$(23)$ & Cohort & 124379 & 11262 & 74133 (59) & 52898 (42.5) & $63.4 \pm 7.2$ & $\mathrm{~N} / \mathrm{A}$ & 98871 (79.5) & 25508 (20.5) \\
\hline$(22)$ & Case-Control & 5063 & 640 & \multicolumn{2}{|c|}{$194(3.8)^{* *}$} & $59.2 \pm 7.9$ & $24.2 \pm 3.3$ & $\mathrm{~N} / \mathrm{A}$ & N/A \\
\hline$(24)$ & Cohort & 3639 & 348 & 95 (2.6) & N/A & $66.9 \pm 9.6$ & $27.0 \pm 4.4$ & 3639 (100) & $0(0)$ \\
\hline$(25)$ & Case-Control & 16299 & 2811 & 369 (2) & 2906 (18) & $63.6 \pm 8.3$ & $24.2 \pm 3.5$ & 16299 (100) & $0(0)$ \\
\hline (26) & Case-Control & 17076 & 1288 & N/A & 3186 (18) & $59.0 \pm 6.7$ & $22.8 \pm 3.4$ & 17076 (100) & $0(0)$ \\
\hline
\end{tabular}


Table 2 Number of participants according to the age of menopause.

\begin{tabular}{|c|c|c|c|c|c|c|c|c|c|c|c|c|}
\hline \multirow[b]{3}{*}{ Study } & \multicolumn{12}{|c|}{ Number of participants according to the age of menopause (years) } \\
\hline & \multicolumn{2}{|c|}{$<40$} & \multicolumn{2}{|c|}{$<45$} & \multicolumn{2}{|c|}{$>45$} & \multicolumn{2}{|c|}{$45-(52-55)$} & \multicolumn{2}{|c|}{$\geq 50$} & \multicolumn{2}{|c|}{$\geq(52-55)$} \\
\hline & Total & T2DM & Total & T2DM & Total & T2DM & Total & T2DM & Total & T2DM & Total & T2DM \\
\hline (13) & 8 & 2 & 50 & 3 & 50 & 3 & 31 & 1 & 19 & 2 & & \\
\hline (11) & 419 & 220 & 1306 & 644 & 6558 & 3047 & 5903 & 2740 & 3988 & 1861 & 655 & 307 \\
\hline$(12)$ & & & 305 & 6 & 4013 & 134 & 3710 & 122 & & & 303 & 12 \\
\hline (14) & & & 671 & 146 & 2633 & 592 & 2022 & 451 & 1820 & 425 & 611 & 141 \\
\hline (18) & & & 660 & 29 & 3756 & 141 & 1290 & 43 & 2466 & 98 & & \\
\hline (19) & 457 & 52 & 865 & 78 & 1732 & 98 & 1551 & 91 & 1037 & 60 & 181 & 7 \\
\hline (20) & & & & & & & & & & & & \\
\hline (21) & & & 351 & 69 & 1753 & 325 & 1464 & 250 & & & 284 & 75 \\
\hline (23) & & & 34922 & 3523 & 89457 & 7739 & 72720 & 6189 & & & 16737 & 1550 \\
\hline (22) & 349 & 40 & 1253 & 142 & 3810 & 498 & 3638 & 461 & 1373 & 198 & 172 & 37 \\
\hline (24) & 83 & 15 & 381 & 54 & 3258 & 294 & 3015 & 280 & 243 & 14 & 243 & 14 \\
\hline (25) & & & 2027 & 401 & 14272 & 2410 & 11863 & 2024 & & & 2409 & 386 \\
\hline (26) & & & 1733 & 125 & 15343 & 1163 & 12991 & 948 & & & 2352 & 215 \\
\hline
\end{tabular}

T2DM, type 2 diabetes mellitus.

\section{Comparison of women with POI with those with normal menopausal age}

Women with POI demonstrated a higher risk for developing T2DM compared with women with normal menopausal age, including those with late menopausal age (five studies, three prospective cohorts) (OR: 1.53, 95\% CI: $\left.1.03-2.27, P=0.035 ; I^{2}: 78 \%, P<0.001\right)(9,11,13$, $19,22)$ (Fig. 4). Compared with women with menopausal age of 45-55 years, women with POI were also at a higher risk of T2DM (five studies, three prospective cohorts) (OR: 1.50, 95\% CI: 1.03-2.19, $\left.\left.P=0.033 ; I^{2}: 75.2 \%, P<0.003\right)\right]$ $(9,11,13,19,22)$ (Fig. 5). The upper limit of normal menopausal age was defined at 49-55 years.

\section{EM vs menopause $>45$ yrs}

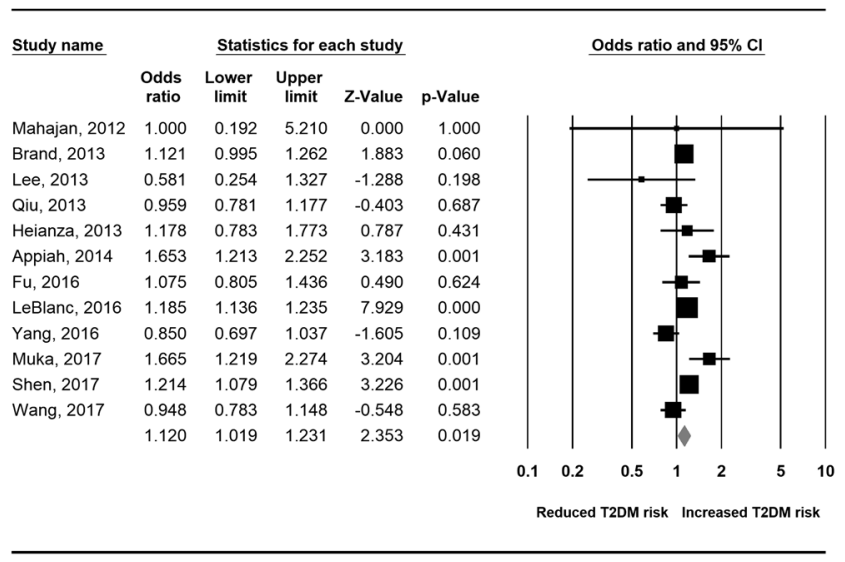

\section{Figure 2}

Forest plot of the comparison between early menopause (EM) and menopause $>45$ years.

\section{Late menopause}

We also investigated for a potential difference between late menopause and normal menopausal age (45-55 years) with respect to T2DM risk (ten studies, four cohorts) $(11,12,14,19,21,22,23,24,25,26)$. A tendency for a higher risk of T2DM was observed for women with late menopausal age (OR: 1.12, 95\% CI: 0.99-1.28, $P=0.069$; $\left.I^{2}: 75.1 \%, P<0.001\right)$.

\section{Meta-regression analysis}

Mean age of the participants at study entry, BMI, smoking status and level of physical activity were used as predictors

\section{EM vs menopause $45-55$ yrs}

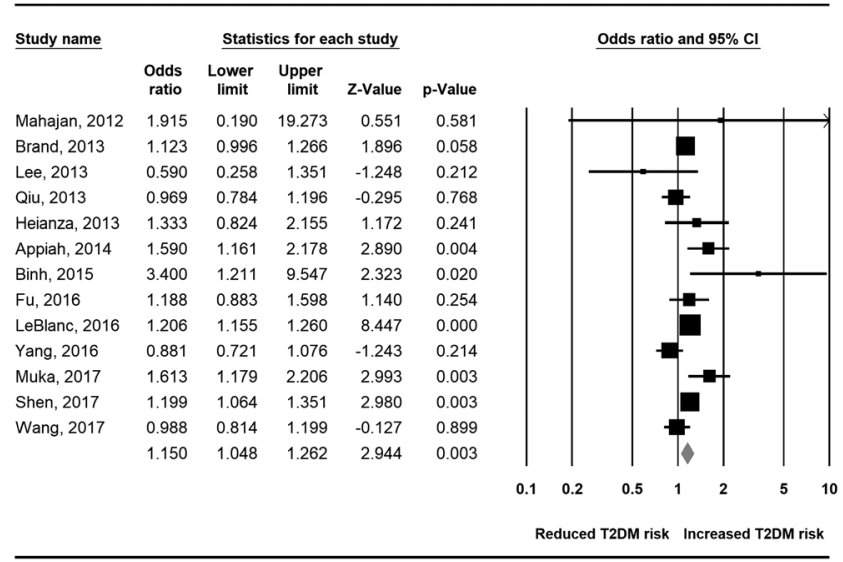

Figure 3

Forest plot of the comparison between early menopause (EM) and menopause at $45-55$ years. 


\section{POI vs menopause $>\mathbf{4 5}$ yrs}

\begin{tabular}{|c|c|c|c|c|c|}
\hline \multirow[t]{2}{*}{ Study name } & \multicolumn{5}{|c|}{ Statistics for each study } \\
\hline & $\begin{array}{c}\text { Odds } \\
\text { ratio }\end{array}$ & $\begin{array}{c}\text { Lower } \\
\text { limit }\end{array}$ & $\begin{array}{c}\text { Upper } \\
\text { limit }\end{array}$ & Z-Value & p-Valu \\
\hline Mahajan, 2012 & 5.222 & 0.721 & 37.850 & 1.636 & 0.102 \\
\hline Brand, 2013 & 1.274 & 1.045 & 1.552 & 2.399 & 0.016 \\
\hline Appiah, 2014 & 2.141 & 1.503 & 3.048 & 4.221 & 0.000 \\
\hline Yang, 2016 & 0.861 & 0.611 & 1.213 & -0.857 & 0.392 \\
\hline Muka, 2017 & 2.224 & 1.255 & 3.940 & 2.740 & 0.006 \\
\hline & 1.531 & 1.031 & 2.273 & 2.111 & 0.035 \\
\hline
\end{tabular}

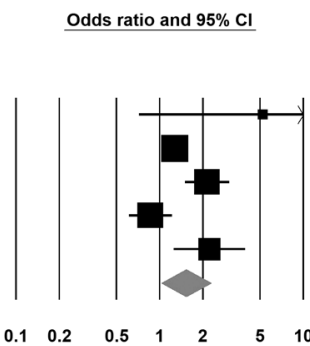

Reduced T2DM risk Increased T2DM risk

\section{Figure 4}

Forest plot of the comparison between premature ovarian insufficiency $(\mathrm{POI})$ and menopause $>45$ years.

of T2DM development. Mean age of the participants appeared to significantly contribute to the impact of the age at menopause on T2DM risk (Q: 7.62, df: $1, P=0.006)$ (Fig. 6). There was no evidence that BMI altered the effect of age at menopause on the risk of T2DM. Meta-regression analysis for smoking status and physical activity was not possible due to the small number of studies or different ways of definition.

\section{Subgroup analysis}

Subgroup analysis was performed with regard to race (Asian vs non-Asian populations), type of menopause (surgical vs natural), study design (cohorts vs case-control studies) and the use of hormone replacement therapy (HRT) and/ or oral contraceptives (OC). All subgroup analyses were

\section{POI vs menopause $45-55$ yrs}

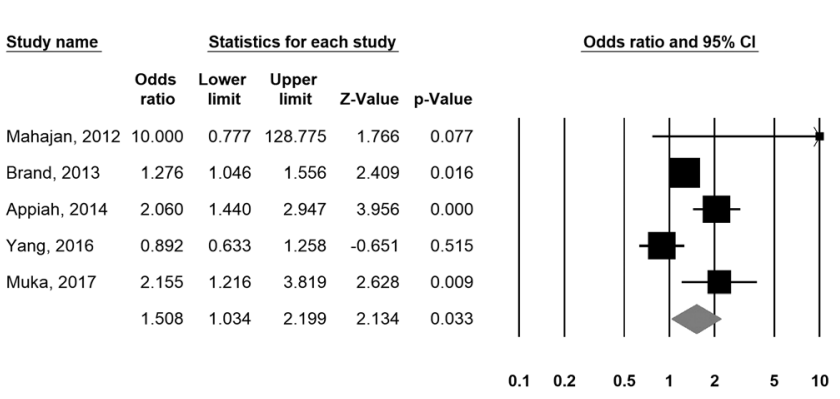

Reduced T2DM risk Increased T2DM risk

\section{Figure 5}

Forest plot of the comparison between premature ovarian insufficiency (POI) and menopause at 45-55 years.

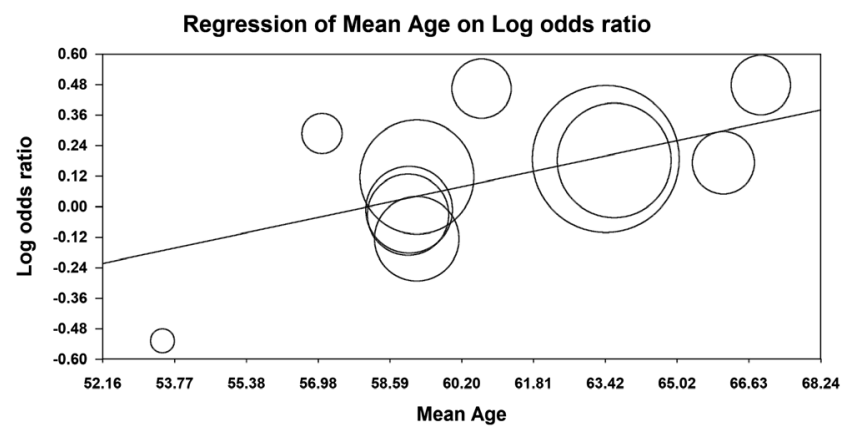

\section{Figure 6}

Meta-regression analysis of the effect of age on the association of premature menopause with increased risk of type 2 diabetes.

performed in the concept of comparison between women with EM and those with natural menopause (age at menopause 45-55 years) (13 studies). Non-Asian women with EM were still at a higher risk of T2DM compared with those with natural menopause of the same race (OR: 1.25, 95\% CI: 1.11-1.41, P<0.001), whereas no such risk was observed for Asian populations (OR: 1.06, 95\% CI: $0.92-1.22, P=0.392)$. However, there was no difference between these two subgroups $(P=0.082)$. The same associations were observed according to the type of study, being significant only for cohort studies $(P<0.001)$, with no significant between-group heterogeneity $(P=0.082)$. Furthermore, no difference was detected according to the use of HRT and/or OC $(P=0.773)$. With respect to the type of menopause, no subgroup analysis could be conducted, since no distinct data on surgical menopause were available.

\section{Discussion}

To the best of our knowledge, this systematic review and meta-analysis including, 191762 postmenopausal women and 21664 cases of T2DM, is the first regarding the association between age of menopause and risk of developing T2DM. Both EM and POI are associated with a higher risk of developing T2DM compared with women with natural menopause, an association that was partially affected by age but not BMI. A tendency for a higher risk was also found for women reporting menopause after 55 years of age.

The exact pathogenetic mechanisms underlying the association between premature menopause and T2DM risk cannot be fully elucidated. A possible mechanism is the shorter exposure to endogenous estrogens, taking into 
consideration the protective role of estrogens on pancreatic $\beta$-cell function and insulin resistance. Estradiol, through binding to its receptor alpha (ER $\alpha)$ in $\beta$-cells and through the concomitant phosphorylation of extracellular signalregulated kinases (ERK1/2), regulates insulin biosynthesis and secretion and modulates $\beta$-cell survival (27). EMor POI-associated glucose intolerance may also be an indirect effect of the metabolic consequences of body fat redistribution due to estrogen deficiency, which leads to increased central adiposity, predisposing to increased insulin resistance $(28,29)$. Moreover, menopauserelated changes in serum concentrations of other steroid hormones, such as testosterone, as well as those of sex hormone-binding globulin (SHBG), may also play a role in the development of T2DM. Testosterone production by the ovaries is reduced to a lesser extent after menopause, compared with that of estradiol (30\% vs $80 \%)(30,31)$. On the other hand, SHBG seems to be protective against T2DM, since its low concentrations have been associated central adiposity and insulin resistance $(30,31)$. Interestingly, a meta-analysis showed that postmenopausal women with T2DM have higher total testosterone and lower SHBG concentrations compared with those without T2DM. In particular, SHBG concentrations $>60 \mathrm{nmol} / \mathrm{L}$ confer a lower risk of T2DM (RR: 0.20, 95\% CI: 0.12-0.30), compared with concentrations $\leq 60 \mathrm{nmol} / \mathrm{L}$ (30).

The main clinical implication of the present study is the identification of women with EM or POI as a group at higher risk of T2DM and at an earlier stage, especially when other risk factors co-exist (e.g. positive family history for T2DM). In these women, an earlier cautiousness and intensification of lifestyle intervention compared with the general population could be of value. Another clinical implication is the beneficial effect of HRT on glucose metabolism in women with EM or POI (32). Data from large interventional studies, such the Women's Health Initiative (WHI) (33) and the Heart and Estrogen/progestin Replacement Study (HERS) (34), have shown a decreased risk of T2DM (21-35\%, after 4.1 or 5.6 years of treatment) with the use of HRT (conjugated estrogen $0.625 \mathrm{mg} /$ day plus medroxyprogesterone acetate $2.5 \mathrm{mg}$ /day) compared to placebo. Notably, this effect was independent of waist circumference or BMI. Current guidelines support a beneficial effect of HRT on T2DM risk, which involves a reduction by $14-19 \%$. This effect does not seem to persist after HRT discontinuation (35).

On the other hand, exposure to excessive estrogen also plays a detrimental role in $\beta$-cell function, since it leads to overstimulation of $\mathrm{ER} \alpha$, resulting in excessive insulin signaling and, eventually, insulin resistance in liver and muscle (27). In a meta-analysis of 13 populationbased prospective studies, high concentrations of total estradiol were associated with increased risk of T2DM (36). This may partly explain the tendency of increased T2DM risk in women with menopausal age $>55$ years, supporting the notion of a U-shape pattern of the effect of estrogen concentrations and duration of exposure on glucose metabolism. Surprisingly, increased body weight seems to be associated with a later age at menopause, which may also explain the tendency of association between late menopause and increased T2DM risk $(37,38)$ The effect of other sex steroid hormones, such as testosterone, on the age at menopause seems to be negligible $(19,36)$, although others have shown an effect of increased androgen concentrations on higher T2DM risk during menopause (39). The aforementioned metaanalysis showed that postmenopausal women with T2DM have higher estradiol concentrations, compared with those without (30) .

Another plausible explanation of the positive association between premature menopause and T2DM could be the co-existence of risk factors that predispose to both conditions, such as smoking, low physical activity, obesity and socioeconomic status. Smoking, a well-known risk factor for T2DM (40), has been also associated with a reduction in time to menopause $(37,38)$, a decrease in size of the ovarian follicle pool (41) and an increase in follicle-stimulating hormone (FSH) concentrations (42). Toxic chemicals released by tobacco use, such as polycyclic aromatic hydrocarbons, may lead to apoptosis of oocytes (43). Unfortunately, we could not perform metaregression analysis on this parameter, due to insufficient and heterogeneous data regarding the definition and classification of smoking habit in the included studies. Furthermore, low socio-economic status and education are associated with both higher prevalence of T2DM (44) and earlier age at menopause (37). Conflicting data exist with regard to physical activity, since low physical activity has been associated with both later (37) and earlier (38) age at menopause.

Genetic variants may predispose to an earlier age of the FMP, since they account for approximately $50 \%$ of the variation in the age at menopause (45). Polymorphisms in genes implicated in DNA repair (such as XO1, HELQ, UIMC1, FAM175A, FANCI, TLK1, POLG, PRIM1) and immune function (such as IL11, NLRP11, BAT2) have been associated with an earlier timing of menopause (46). Whether these polymorphisms predispose to T2DM risk or just to premature aging is unknown. Some DNA repair gene polymorphisms, such as Xeroderma pigmentosum 
complementation group D (XPD) and human oxoguanine glycosylase 1 (hOGG1), have been recognized as potential mediators in the pathogenesis of T2DM (47). Mitochondrial dysfunction seems to play a role both in the timing of menopause (46) and the pathogenesis of T2DM (48). The obscure contribution of a potential genetic predisposition in earlier menopausal age is further supported by the fact that both race and ethnicity do not seem to be significantly associated with the age of menopause in other studies (37). In general, age at the natural menopause is a result of constellation of both genetic and environmental factors (37) and clear causality cannot be supported by the aforementioned associations. The tendency for an association between late menopause and increased T2DM risk may be partly attributed to agerelated metabolic disturbances, such as central obesity and insulin resistance.

The present study has certain limitations. First, the different design of the original studies might have contributed to the relatively high degree of heterogeneity. The main drawback with case-control studies is that they could not ascertain T2DM, the latter being a selfreported diagnosis. However, the meta-analysis includes four cohort-studies, with a follow-up time between 8 and 10 years; the significance in the results remained after performing a subgroup analysis. Second, the diagnosis of T2DM was set with different methodology in the studies included. However, after performing a separate analysis for the studies that used either FPG, OGTT or $\mathrm{HbA}_{1 \mathrm{c}}$ concentrations, the association between EM and T2DM remained significant (eight studies); the same was not true for the association between POI and T2DM (two studies). Third, past use of HRT or OC was not reported in five studies. Of note, the percentage of the participants having received HRT or OC was relatively low and the exact duration could not be defined in most studies. Therefore, it is probable that it did not alter the results, taking also into account the fact that in the remaining eight studies, subgroup analyses did not demonstrate such an effect. Fourth, in most subjects included, the age of natural menopause was self-reported (defined as 12 months of amenorrhea), which was subjected to recall bias and the nature of cross-sectional design. Moreover, the cause of EM, which may include chemoradiation or oophorectomy, may have had an independent impact on the risk of T2DM. Unfortunately, no data were available on the exact cause of EM and its impact on T2DM. Therefore, no subgroup analysis could be performed on this regard. Fifth, we did not use (age-adjusted or unadjusted) OR directly from the included studies. Only absolute numerical data were extracted, except for the study by Binh et al. (20), to perform comparisons between T2DM and non-T2DM cases in women with EM and normal menopause.

\section{Conclusions}

In summary, this systematic review and meta-analysis in postmenopausal women shows that women entering menopause at an earlier age (either $<45$ or $<40$ years) have an increased risk of developing T2DM compared with those with menopausal age $>45$ years. This should be taken into account in building prognostic models to early detection of T2DM in women, especially in those at highrisk status, to necessitate lifestyle intervention strategies and potential pharmaceutical therapy. Well-designed, prospective cohort and interventional studies will further elucidate these issues.

\section{Supplementary data}

This is linked to the online version of the paper at https://doi.org/10.1530/ EJE-18-0602.

\section{Declaration of interest}

Dr Stevenson has received grants/research support from Abbott, Mylan and Pfizer; consulting fees from Abbott, Mylan and Pfizer and speaker's honoraria from Abbott, Bayer, Gedeon Richter, Menarini, Mylan and Pfizer. The other authors declare that there is no conflict of interest that could be perceived as prejudicing the impartiality of this study.

\section{Funding}

This research did not receive any specific grant from any funding agency in the public, commercial or not-for-profit sector.

\section{Author contribution statement}

$\mathrm{P}$ A designed the research, extracted and analyzed the data and wrote the first draft of the paper. K C, A-M A, N K G, N K and P S searched the literature, extracted and analyzed the data. $K C$ was responsible for the statistical analysis and reviewed the manuscript. S A P, M P, E T, E K, I L and J C S reviewed the manuscript and provided critical scientific input. D $\mathrm{G} G$ resolved discrepancies regarding the quality of the studies included in the meta-analysis, provided critical scientific input and had the primary responsibility for the paper's final content.

\section{References}

1 Davis SR, Lambrinoudaki I, Lumsden M, Mishra GD, Pal L, Rees M, Santoro N \& Simoncini T. Menopause. Nature Reviews. Disease Primers 20151 15004. (https://doi.org/10.1038/nrdp.2015.4)

2 Richardson SJ, Senikas V \& Nelson JF. Follicular depletion during the menopausal transition: evidence for accelerated loss and ultimate 
exhaustion. Journal of Clinical Endocrinology and Metabolism 198765 1231-1237. (https://doi.org/10.1210/jcem-65-6-1231)

3 Cooper GS \& Sandler DP. Age at natural menopause and mortality. Annals of Epidemiology 19988 229-235. (https://doi.org/10.1016/ S1047-2797(97)00207-X)

4 Luborsky JL, Meyer P, Sowers MF, Gold EB \& Santoro N. Premature menopause in a multi-ethnic population study of the menopause transition. Human Reproduction 200318 199-206. (https://doi. org/10.1093/humrep/deg005)

5 Anagnostis P, Stevenson JC, Crook D, Johnston DG \& Godsland IF. Effects of menopause, gender and age on lipids and high-density lipoprotein cholesterol subfractions. Maturitas 201581 62-68. (https://doi.org/10.1016/j.maturitas.2015.02.262)

6 Anagnostis P, Stevenson JC, Crook D, Johnston DG \& Godsland IF. Effects of gender, age and menopausal status on serum apolipoprotein concentrations. Clinical Endocrinology 201685 733-740. (https://doi.org/10.1111/cen.13085)

7 Stefanska A, Bergmann K \& Sypniewska G. Metabolic syndrome and menopause: pathophysiology, clinical and diagnostic significance. Advances in Clinical Chemistry 201572 1-75.

8 Leeners B, Geary N, Tobler PN \& Asarian L. Ovarian hormones and obesity. Human Reproduction Update 201723 300-321. (https://doi. org/10.1093/humupd/dmw045)

9 Muka T, Oliver-Williams C, Kunutsor S, Laven JS, Fauser BC, Chowdhury R, Kavousi M \& Franco OH. Association of age at onset of menopause and time since onset of menopause with cardiovascular outcomes, intermediate vascular traits, and all-cause mortality: a systematic review and meta-analysis. JAMA Cardiology 20161 767-776. (https://doi.org/10.1001/jamacardio.2016.2415)

10 Tao XY, Zuo AZ, Wang JQ \& Tao FB. Effect of primary ovarian insufficiency and early natural menopause on mortality: a metaanalysis. Climacteric 201619 27-36. (https://doi.org/10.3109/136971 37.2015.1094784)

11 Brand JS, Van Der Schouw YT, Onland-Moret NC, Sharp SJ, Ong KK, Khaw KT, Ardanaz E, Amiano P, Boeing H, Chirlaque MD et al. Age at menopause, reproductive life span, and type 2 diabetes risk: Results from the EPIC-InterAct study. Diabetes Care 201336 1012-1019. (https://doi.org/10.2337/dc12-1020)

12 Lee JS, Hayashi K, Mishra G, Yasui T, Kubota T \& Mizunuma H. Independent association between age at natural menopause and hypercholesterolemia, hypertension, and diabetes mellitus: Japan nurses' health study. Journal of Atherosclerosis and Thrombosis 201320 161-169. (https://doi.org/10.5551/jat.14746)

13 Mahajan N, Aggarwal M \& Bagga A. Health issues of menopausal women in North India. Journal of Mid-life Health 20123 84-87. (https://doi.org/10.4103/0976-7800.104467)

14 Qiu C, Chen H, Wen J, Zhu P, Lin F, Huang B, Wu P, Lin Q, Lin Y, $\mathrm{Rao} \mathrm{H}$ et al. Associations between age at menarche and menopause with cardiovascular disease, diabetes, and osteoporosis in Chinese Women. Journal of Clinical Endocrinology and Metabolism 201398 1612-1621. (https://doi.org/10.1210/jc.2012-2919)

15 Anagnostis P, Majeed A, Johnston DG \& Godsland IF. Cardiovascular risk in women with type 2 diabetes mellitus and prediabetes: is it indeed higher than men? European Journal of Endocrinology 2014171 R245-R255. (https://doi.org/10.1530/EJE-14-0401)

16 Liberati A, Altman DG, Tetzlaff J, Mulrow C, Gotzsche PC, Ioannidis JP, Clarke M, Devereaux PJ, Kleijnen J \& Moher D. The PRISMA statement for reporting systematic reviews and meta-analyses of studies that evaluate healthcare interventions: explanation and elaboration. BMJ 2009339 b2700. (https://doi. org/10.1136/bmj.b2700)

17 Wells GA SB, O'Connell D, Peterson J, Welch V \& Tugwell P. The Newcastle-Ottawa Scale (NOS) for assessing the quality of nonrandomised studies in meta-analyses. 3rd Symposium on Systematic Reviews: Beyond the Basics.
18 Heianza Y, Arase Y, Kodama S, Hsieh SD, Tsuji H, Saito K, Shimano H, Hara S \& Sone H. Effect of postmenopausal status and age at menopause on type 2 diabetes and prediabetes in Japanese individuals: Toranomon Hospital Health Management Center study 17 (TOPICS 17). Diabetes Care 201336 4007-4014. (https://doi. org/10.2337/dc13-1048)

19 Appiah D, Winters SJ \& Hornung CA. Bilateral oophorectomy and the risk of incident diabetes in postmenopausal women. Diabetes Care 201437 725-733. (https://doi.org/10.2337/dc13-1986)

20 Binh TQ \& Nhung BT. Prevalence and risk factors of type 2 diabetes in middle-aged women in Northern Vietnam. International Journal of Diabetes in Developing Countries 201636 150-157. (https://doi. org/10.1007/s13410-015-0372-6)

21 Fu Y, Yu Y, Wang S, Kanu JS, You Y, Liu Y, Zhang Y, Liu Y, Li B, Tao Y et al. Menopausal age and chronic diseases in elderly women: a cross-sectional study in Northeast China. International Journal of Environmental Research and Public Health 201613 E396. (https://doi. org/10.3390/ijerph13100936)

22 Yang A, Liu S, Cheng N, Pu H, Dai M, Ding J, Li J, Li H, Hu X, Ren $\mathrm{X}$ et al. Reproductive factors and risk of type 2 diabetes in an occupational cohort of Chinese women. Journal of Diabetes and Its Complications 201630 1217-1222. (https://doi.org/10.1016/j. jdiacomp.2016.06.011)

23 LeBlanc ES, Kapphahn K, Hedlin H, Desai M, Parikh NI, Liu S, Parker DR, Anderson M, Aroda V, Sullivan S et al. Reproductive history and risk of type 2 diabetes mellitus in postmenopausal women: Findings from the Women's Health Initiative. Menopause 201724 64-72. (https://doi.org/10.1097/GME.0000000000000714)

24 Muka T, Asllanaj E, Avazverdi N, Jaspers L, Stringa N, Milic J, Ligthart S, Ikram MA, Laven JSE, Kavousi M et al. Age at natural menopause and risk of type 2 diabetes: a prospective cohort study. Diabetologia 201760 1951-1960. (https://doi.org/10.1007/s00125017-4346-8)

25 Shen L, Song L, Li H, Liu B, Zheng X, Zhang L, Yuan J, Liang Y \& Wang Y. Association between earlier age at natural menopause and risk of diabetes in middle-aged and older Chinese women: The DongfengTongji cohort study. Diabetes and Metabolism 201743 345-350.

26 Wang M, Hu RY, Wang H, Gong WW, Wang CM, Xie KX, Chen ZM, Guo Y, Yu M \& Li LM. Age at natural menopause and risk of diabetes in adult women: findings from the China Kadoorie Biobank study in Zhejiang area. Journal of Diabetes Investigation 20189 762-768. (https://doi.org/10.1111/jdi.12775)

27 Nadal A, Alonso-Magdalena P, Soriano S, Quesada I \& Ropero AB. The pancreatic beta-cell as a target of estrogens and xenoestrogens: Implications for blood glucose homeostasis and diabetes. Molecular and Cellular Endocrinology 2009304 63-68. (https://doi.org/10.1016/j. mce.2009.02.016)

28 Paschou SA, Anagnostis P, Pavlou DI, Vryonidou A, Goulis DG \& Lambrinoudaki I. Diabetes in menopause: risks and management. Current Vascular Pharmacology 2018 Epub. (https://doi.org/10.2174/15 $70161116666180625124405)$

29 Carr MC. The emergence of the metabolic syndrome with menopause. Journal of Clinical Endocrinology and Metabolism $2003 \mathbf{8 8}$ 2404-2411. (https://doi.org/10.1210/jc.2003-030242)

30 Ding EL, Song Y, Malik VS \& Liu S. Sex differences of endogenous sex hormones and risk of type 2 diabetes: a systematic review and meta-analysis. JAMA 2006295 1288-1299. (https://doi.org/10.1001/ jama.295.11.1288)

31 Vryonidou A, Paschou SA, Muscogiuri G, Orio F \& Goulis DG. MECHANISMS IN ENDOCRINOLOGY: Metabolic syndrome through the female life cycle. European Journal of Endocrinology 2015173 R153-R163. (https://doi.org/10.1530/EJE-15-0275)

32 Slopien R, Wender-Ozegowska E, Rogowicz-Frontczak A, Meczekalski B, Zozulinska-Ziolkiewicz D, Jaremek JD, Cano A, Chedraui P, Goulis DG, Lopes P et al. Menopause and diabetes: EMAS 
clinical guide. Maturitas 2018117 6-10. (https://doi.org/10.1016/j. maturitas.2018.08.009)

33 Margolis KL, Bonds DE, Rodabough RJ, Tinker L, Phillips LS, Allen C, Bassford T, Burke G, Torrens J \& Howard BV. Effect of oestrogen plus progestin on the incidence of diabetes in postmenopausal women: results from the Women's Health Initiative Hormone Trial. Diabetologia 200447 1175-1187. (https://doi.org/10.1007/s00125004-1448-x)

34 Kanaya AM, Herrington D, Vittinghoff E, Lin F, Grady D, Bittner V, Cauley JA \& Barrett-Connor E. Glycemic effects of postmenopausal hormone therapy: the Heart and Estrogen/progestin Replacement Study. A randomized, double-blind, placebo-controlled trial. Annals of Internal Medicine 2003138 1-9. (https://doi.org/10.7326/00034819-138-1-200301070-00005)

35 Stuenkel CA, Davis SR, Gompel A, Lumsden MA, Murad MH, Pinkerton JV \& Santen RJ. Treatment of symptoms of the menopause: an Endocrine Society Clinical Practice Guideline. Journal of Clinical Endocrinology and Metabolism 2015100 3975-4011. (https://doi.org/10.1210/jc.2015-2236)

36 Muka T, Nano J, Jaspers L, Meun C, Bramer WM, Hofman A, Dehghan A, Kavousi M, Laven JS \& Franco OH. Associations of steroid sex hormones and sex hormone-binding globulin with the risk of type 2 diabetes in women: a population-based cohort study and meta-analysis. Diabetes 201766 577-586. (https://doi. org/10.2337/db16-0473)

37 Gold EB, Crawford SL, Avis NE, Crandall CJ, Matthews KA, Waetjen LE, Lee JS, Thurston R, Vuga M \& Harlow SD. Factors related to age at natural menopause: longitudinal analyses from SWAN. American Journal of Epidemiology 2013178 70-83. (https://doi. org/10.1093/aje/kws421)

38 Stepaniak U, Szafraniec K, Kubinova R, Malyutina S, Peasey A, Pikhart H, Pajak A \& Bobak M. Age at natural menopause in three central and eastern European urban populations: the HAPIEE study. Maturitas 201375 87-93. (https://doi.org/10.1016/j. maturitas.2013.02.008)

39 Ding EL, Song Y, Manson JE, Rifai N, Buring JE \& Liu S. Plasma sex steroid hormones and risk of developing type 2 diabetes in women: a prospective study. Diabetologia 200750 2076-2084. (https://doi. org/10.1007/s00125-007-0785-y)

40 Chang SA. Smoking and type 2 diabetes mellitus. Diabetes and Metabolism Journal 201236 399-403.

41 Westhoff C, Murphy P \& Heller D. Predictors of ovarian follicle number. Fertility and Sterility $2000 \mathbf{7 4}$ 624-628. (https://doi. org/10.1016/S0015-0282(00)01527-2)

42 Cooper GS, Baird DD, Hulka BS, Weinberg CR, Savitz DA \& Hughes CL Jr Follicle-stimulating hormone concentrations in relation to active and passive smoking. Obstetrics and Gynecology 1995 85 407-411. (https://doi.org/10.1016/0029-7844(94)00381-M)

43 Matikainen T, Perez GI, Jurisicova A, Pru JK, Schlezinger JJ, Ryu HY, Laine J, Sakai T, Korsmeyer SJ, Casper RF et al. Aromatic hydrocarbon receptor-driven Bax gene expression is required for premature ovarian failure caused by biohazardous environmental chemicals. Nature Genetics 200128 355-360. (https://doi.org/10.1038/ng575)

44 Rabi DM, Edwards AL, Southern DA, Svenson LW, Sargious PM, Norton P, Larsen ET \& Ghali WA. Association of socio-economic status with diabetes prevalence and utilization of diabetes care services. BMC Health Services Research 20066 124. (https://doi. org/10.1186/1472-6963-6-124)

45 Laven JS. Genetics of early and normal menopause. Seminars in Reproductive Medicine 201533 377-383. (https://doi. org/10.1055/s-0035-1567825)

46 Stolk L, Perry JR, Chasman DI, He C, Mangino M, Sulem P, Barbalic M, Broer L, Byrne EM, Ernst F et al. Meta-analyses identify 13 loci associated with age at menopause and highlight DNA repair and immune pathways. Nature Genetics 201244 260-268. (https:// doi.org/10.1038/ng.1051)

47 Kasznicki J, Krupa R, Blasiak J \& Drzewoski J. Association between polymorphisms of the DNA repair genes XRCC1 and hOGG1 and type 2 diabetes mellitus in the Polish population. Polskie Archiwum Medycyny Wewnetrznej 2009119 122-128.

48 Rolo AP \& Palmeira CM. Diabetes and mitochondrial function: role of hyperglycemia and oxidative stress. Toxicology and Applied Pharmacology 2006212 167-178. (https://doi.org/10.1016/j. taap.2006.01.003)

Received 16 July 2018

Revised version received 14 September 2018

Accepted 25 October 2018 